\title{
Electron optics in phosphorene pn junctions: Negative reflection and anti super-Klein tunneling
}

\author{
Yonatan Betancur-Ocampo, ${ }^{1,}$ 用 François Leyvraz, ${ }^{1,2}$ and Thomas Stegmann ${ }^{1, \dagger}$ \\ ${ }^{1}$ Instituto de Ciencias Físicas, Universidad Nacional Autónoma de México, Cuernavaca, México \\ ${ }^{2}$ Centro Internacional de Ciencias, Cuernavaca, México
}

\begin{abstract}
Ballistic electrons in phosphorene pn junctions show optical-like phenomena. Phosphorene is modeled by a tight-binding Hamiltonian that describes its electronic structure at low energies, where the electrons behave in the zigzag direction as massive Dirac fermions and in the orthogonal armchair direction as Schrödinger electrons. Applying the continuum approximation, we derive the electron optics laws in phosphorene $p n$ junctions, which show very particular and unusual properties. Due to the anisotropy of the electronic structure, these laws depend strongly on the orientation of the junction with respect to the sublattice. Negative and anomalous reflection are observed for tilted junctions, while the typical specular reflection is found only, if the junction is parallel to the zigzag or armchair edges. Moreover, omni-directional total reflection, called anti-super Klein tunneling, is observed if the junction is parallel to the armchair edge. Applying the nonequilibrium Green's function method on the tight-binding model, we calculate numerically the current flow. The good agreement of both approaches confirms the atypical transport properties, which can be used in nano-devices to collimate and filter the electron flow, or to switch its direction.
\end{abstract}

\section{INTRODUCTION}

Electron optics originates from the wave-particle duality and has lead to outstanding technological applications like the electron microscope [1]. The rise of graphene and other two-dimensional materials has given electron optics recently a new turn, because ballistic ray-like electron propagation can be observed in these materials at low energies. The electron rays can be manipulated by external gates that form a $p n$ junction [2 5]. In graphene, the electrons are refracted at the interface of the junction following a generalized Snell's law, where the gate voltages in the different regions play the role of the refractive indices and cause negative refraction [2]. Moreover, perfect transmission at normal incidence can be observed, which is known as Klein tunneling 6. These phenomena have been confirmed experimentally $7-9$. Another recently discussed possibility to manipulate the electron flow in graphene is to deform the material elastically [10 16]. These discoveries made it possible to propose new nano-devices such as superlenses [2, 12, 17, valley beam splitters [10, 11, 18 26] and collimators [5, 10, 27].

Recently, a monolayer of black phosphorous called phosphorene has been synthesized [28. Also phosphorene nanoribbons have been produced 29]. The electronic structure of this material shows (in contrast to graphene) an intrinsic band-gap and strong anisotropy, which causes the electrons to behave in one direction like massive Dirac fermions and in the orthogonal direction like non-relativistic Schrödinger electrons [30 45]. This unique electronic structure makes phosphorene very attractive for both, fundamental research and technological applications such as transistors [46 53] and sensors [54].

* ybetancur@icf.unam.mx

$\dagger$ stegmann@icf.unam.mx
In this article, we study theoretically the current flow in phosphorene $p n$ junctions and answer the question how electron beams are reflected and transmitted at the interface of the junction. We start from a simple second nearest neighbor tight-binding model that reproduces well the electronic structure of phosphorene at low energies. Using this Hamiltonian, on the one hand, we calculate numerically the current flow by means of the nonequilibrium Green's function (NEGF) method. On the other hand, applying the continuum approximation, we calculate the semiclassical trajectories of the electrons and derive the laws of reflection and refraction for electron optics in phosphorene $p n$ junctions. Both approaches agree qualitatively and demonstrate unusual optical phenomena like negative and anomalous reflection, which have been observed previously only for electromagnetic waves at the surface of meta materials [55-58. For certain system parameters we also report omni-directional perfect reflection, called anti super-Klein tunneling, which is the counterpart of the omnidirectional perfect transmission of massless Dirac fermions in Dirac materials [17] and pseudo-spin one systems [5962]. Our theoretically work will pave the way to electron optics experiments in phosphorene, which to the best of our knowledge have not yet been performed.

\section{MODELING PHOSPHORENE}

Phosphorene is a two-dimensional puckered crystal with four phosphorous atoms in the unit cell, see Figure 1 (a). A detailed introduction and an overview can be found in Refs. 31, 35 39. The electronic structure of phosphorene is modeled by the tight-binding Hamiltonian

$$
H=\sum_{\langle i, j\rangle} t_{i j}|i\rangle\langle j|+\text { H.c. }
$$


(a)

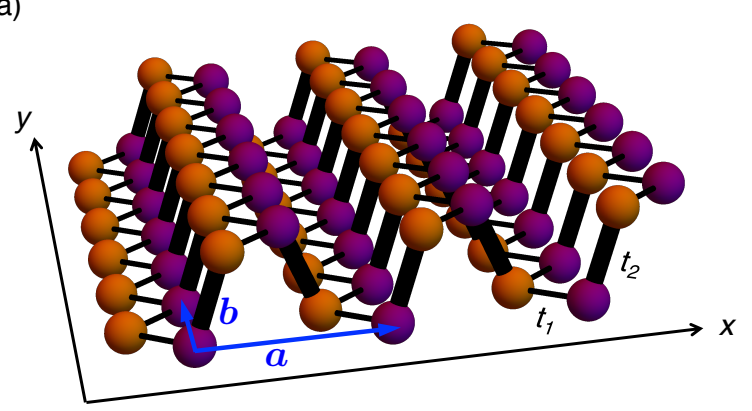

(b)

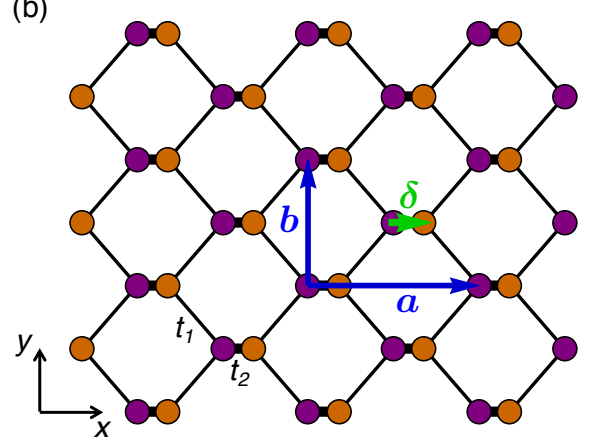

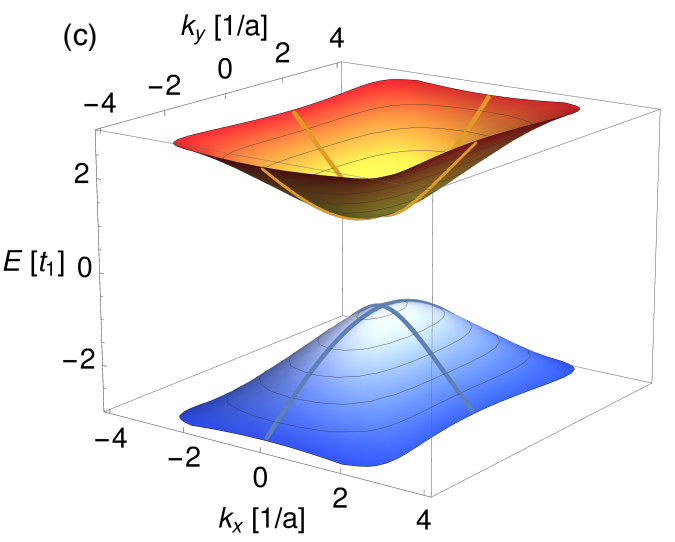

(d)

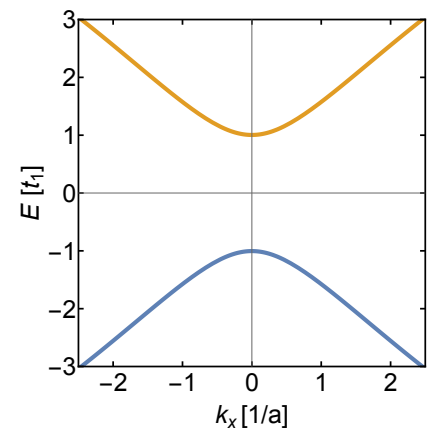

(e)

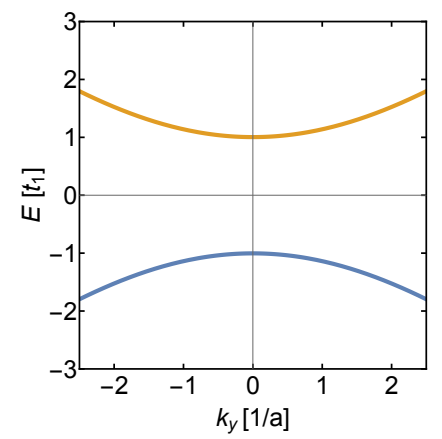

Figure 1. (a) Crystal structure of phosphorene. Its electronic structure is modeled by a tight-binding Hamiltonian that takes into account first $\left(t_{1}\right)$ and second $\left(t_{2} \approx-3 t_{1}\right)$ nearest neighbors. The vectors $\boldsymbol{a}$ and $\boldsymbol{b}$ span the part of the unit cell that is in the $x y$ plane. (b) Projection of all atoms to the $x y$ plane shows that phosphorene can be also understood as a deformed trigonal lattice with a diatomic basis, as indicated by the different coloring of the atoms. The vector $\boldsymbol{\delta}$ connects these two atoms of the unit cell. (c) The electronic band structure of phosphorene shows a band gap at the $\Gamma$ point and is highly anisotropic. The approximately linear dispersion of massive Dirac fermions is observed in the $k_{x}$ direction (d), while the parabolic dispersion of Schrödinger electrons is found along the $k_{y}$ direction (e).

where the $|i\rangle$ represent the atomic states localized on the phosphorous atoms at positions $\boldsymbol{r}_{i}$. The sum includes first and second nearest neighbors which are coupled by the energies $t_{1}=-1.22 \mathrm{eV}$ and $t_{2}=3.665 \mathrm{eV}$, respectively [41, 42].

The physical description of phosphorene can be simplified by projecting all atoms on the $x y$ plane keeping the coupling energies constant [21, 35, 37]. After this projection, see Figure 1(b), the phosphorene lattice can be understood as a strongly deformed honeycomb lattice, where the armchair edge runs along the $x$ direction while the zigzag boundary is parallel to the $y$ direction. This lattice is formed by trigonal basis vectors and has a unit cell with only two atoms that are connected by the vector $\boldsymbol{\delta}$ of length $0.8 \AA$. From this point of view phosphorene is very similar to deformed graphene. However, the clear difference between the hopping parameters $\left(t_{2} \approx-3 t_{1}\right)$ causes very different physical properties. For simplicity we will continue using the orthogonal basis vectors of the original phosphorene lattice, $\boldsymbol{a}$ and $\boldsymbol{b}$, which have a length of $4.42 \AA$ and $3.27 \AA$, respectively [40].

Making a plane wave ansatz, the tight-binding Hamil- tonian can be written as

$$
H(\boldsymbol{k})=\left(\begin{array}{cc}
0 & g^{*}(\boldsymbol{k}) \\
g(\boldsymbol{k}) & 0
\end{array}\right)
$$

where

$$
g(\boldsymbol{k})=\mathrm{e}^{-\mathrm{i} k_{\delta}}\left[t_{2}+2 t_{1} \mathrm{e}^{\mathrm{i} k_{a} / 2} \cos \left(k_{b} / 2\right)\right]
$$

with $k_{\delta}=\boldsymbol{k} \cdot \boldsymbol{\delta}, k_{a}=\boldsymbol{k} \cdot \boldsymbol{a}$ and $k_{b}=\boldsymbol{k} \cdot \boldsymbol{b}$. The energy bands of phosphorene are given by

$$
\begin{gathered}
E(\boldsymbol{k})=s|g(\boldsymbol{k})| \\
=s \sqrt{t_{2}^{2}+4 t_{1} t_{2} \cos \left(k_{a} / 2\right) \cos \left(k_{b} / 2\right)+4 t_{1}^{2} \cos ^{2}\left(k_{b} / 2\right)}
\end{gathered}
$$

where $s=\operatorname{sgn}(E)$ is the band index. The energy bands, see Figure 1 (c)-(e), show a direct band gap of $2 \Delta=$ $4 t_{1}+2 t_{2}$ in the center of the Brillouin zone ( $\Gamma$ point) and are highly anisotropic. The corresponding eigenfunctions read

$$
\Psi_{\boldsymbol{k}}(\boldsymbol{r})=\frac{1}{\sqrt{2}}\left(\begin{array}{c}
1 \\
s \mathrm{e}^{\mathrm{i} \phi(\boldsymbol{k})}
\end{array}\right) \mathrm{e}^{\mathrm{i} \boldsymbol{k} \cdot \boldsymbol{r}},
$$


(a)

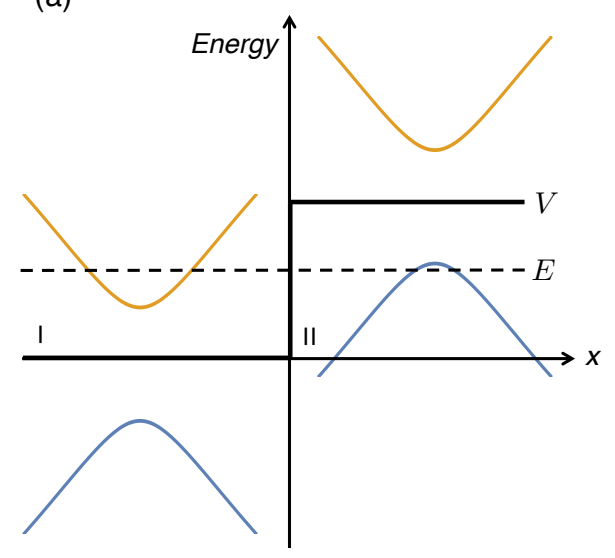

(b)

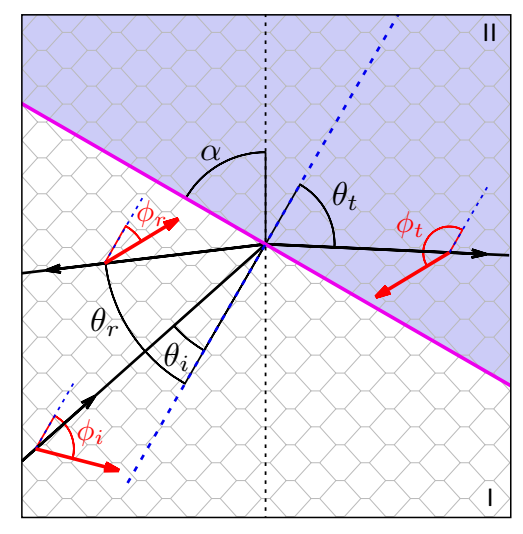

(c)

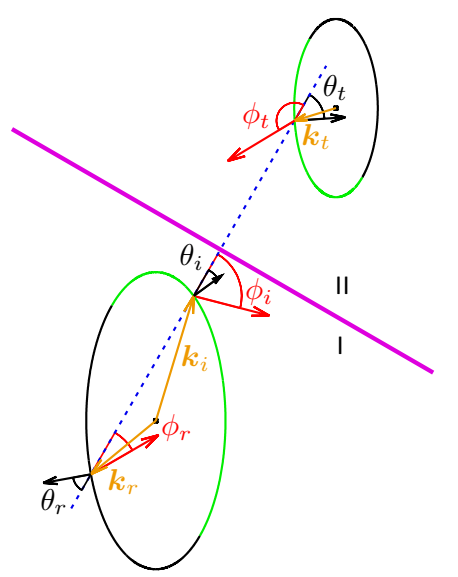

Figure 2. Electron optics in a phosphorene $p n$ junction. (a) The gate-induced electrostatic potential $V$ shifts the energy bands of phosphorene and generates a $p n$ junction. In the left region I the electrons of energy $E$ belong to the conduction band (orange), while in the right region II they are in the valance band (blue). (b) The interface of the junction (violet solid line) is tilted by the angle $\alpha$, which is an important parameter due to the anisotropy of phosphorene's electronic structure. An incident electron beam (black solid line) with pseudo-spin $\phi_{i}$ (red arrow) hits the interface under the angle $\theta_{i}$. It is reflected (atypically) under the angle $\theta_{r}$ with pseudo-spin $\phi_{r}$, while it is transmitted under the angle $\theta_{t}$ with pseudo-spin $\phi_{t}$. (c) Kinematical construction in the momentum space. The closed curves are constant energy contours. The current density (or group velocity) vectors are represented by the black arrows, which are orthogonal to the energy contours. The green semi-arcs indicate the regions where the current density $j_{\perp}$ is conserved. The blue-dashed line represents the conservation of the parallel momentum $k_{\|}$. Its intersection points with the constant energy contours determine the reflected and transmitted states (orange arrows).

with the phase (or pseudo-spin)

$$
\begin{aligned}
\phi(\boldsymbol{k}) & =\arctan \left(\frac{\operatorname{Im}[g(\boldsymbol{k})]}{\operatorname{Re}[g(\boldsymbol{k})]}\right) \\
& =\arctan \left(\frac{\lambda_{1}(\boldsymbol{k}) \cos \left(k_{\delta}\right)-\lambda_{2}(\boldsymbol{k}) \sin \left(k_{\delta}\right)}{\lambda_{2}(\boldsymbol{k}) \cos \left(k_{\delta}\right)+\lambda_{1}(\boldsymbol{k}) \sin \left(k_{\delta}\right)}\right),
\end{aligned}
$$

which is a function of $\lambda_{1}(\boldsymbol{k})=2 t_{1} \sin \left(k_{a} / 2\right) \cos \left(k_{b} / 2\right)$ and $\lambda_{2}(\boldsymbol{k})=t_{2}+2 t_{1} \cos \left(k_{a} / 2\right) \cos \left(k_{b} / 2\right)$. Note that the prefactor $\mathrm{e}^{-\mathrm{i} k_{\delta}}$ in $(3)$ is frequently omitted because it cancels out in the calculation of the energy bands. However, the electron propagation will be described incorrectly if this term is not included in the eigenfunctions, because their phase depends explicitly on $k_{\delta}$.

The anisotropy of the energy bands of phosphorene is observed more clearly, if the Hamiltonian (2) is expanded around the $\Gamma$ point ${ }^{1}$

$$
H_{\mathrm{eff}}=\sigma_{x}\left(\Delta+\frac{p_{x}^{2}}{2 m_{x}}+\frac{p_{y}^{2}}{2 m_{y}}\right)+\sigma_{y} v p_{x},
$$

where $m_{x}=2 /\left(-t_{1} a^{2}+2 \delta\left(2 a t_{1}-\delta \Delta\right)\right)$ and $m_{y}=$ $-2 /\left(t_{1} b^{2}\right)$ are the anisotropic masses, and $v=a t_{1}-\delta \Delta$

\footnotetext{
${ }^{1}$ Note that in contrast to graphene, where the Dirac cones are located at the six $K$ points, the band gap in phosphorene appears at the single $\Gamma$ point in the center of the Brillouin zone.
}

is the velocity along the $x$ direction. The energy bands take the form

$$
E_{s}=s \sqrt{\left(\Delta+\frac{p_{x}^{2}}{2 m_{x}}+\frac{p_{y}^{2}}{2 m_{y}}\right)^{2}+v^{2} p_{x}^{2}}
$$

and show clearly the hybrid behavior of the electrons in the different directions. If $p_{y}=0$, the electrons behave approximately as massive Dirac fermions with velocity $v_{F}=\sqrt{v^{2}+\Delta / m_{x}}$ and rest mass $m_{0}=\Delta / v_{F}^{2}$. If $p_{x}=0$, the electrons have the parabolic dispersion of Schrödinger electrons.

To conclude this section, the electronic band structure in Figure 1 (c)-(e) shows that the used second nearest neighbor tight-binding model reproduces well the essential electronic properties of phosphorene at low energies, namely a band gap and strong anisotropy. Taking into account higher orders modify the size of the band gap, but will not change qualitatively the shape of the electronic structure (at low energies), which is the basis for electron optics to be discussed in the following sections.

\section{ELECTRON OPTICS IN PHOSPHORENE PN JUNCTIONS}

A phosphorene $p n$ junction is constituted by a monolayer of black phosphorous with two differently doped regions, I and II, respectively. These regions, which are indicated in Figure 2 (b) by different background color 
shadings, are realized experimentally by gates that induce different electrostatic potentials. These potentials shift the electronic structure and hence, change the electron densities, see Figure 2 (a). Without loss of generality, we assume that the electrostatic potential is zero in the white-shaded region I, where the electrons will be injected, while it has the value $V$ in the blue-shaded region II. At the interface between the regions the electrons are reflected and refracted in different directions, similar to a light beam that passes from one medium to another. Using the continuum approximation of phosphorene, we consider the interface as a straight line without any corrugation due to the lattice structure. Moreover, we assume that at the interface the potential changes abruptly on the length scale defined by the Fermi wavelength $\lambda_{F}=2 \pi /|\boldsymbol{k}|$. This assumption is made because the band gap of phosphorene damps drastically the transmission through a junction with a smooth potential profile.

As phosphorene is a material with an anisotropic electronic structure, the transport properties through the $p n$ junction depend on the orientation of the junction with respect to the phosphorene lattice, which is measured in the following by the angle $\alpha$. The incident electron beam with energy $E$ hits the interface of the junction under the angle $\theta_{i}$. These four parameters, $V, \alpha, E, \theta_{i}$, which have a clear physical meaning, will be used in the following to characterize the system. All other quantities will be calculated as a function of these parameters. For example, the wave vector $\boldsymbol{k}_{i}$ of the incident electrons is calculated by using (4) to obtain

$$
k_{a}=2 \arccos \left(\frac{E^{2}-t_{2}^{2}-4 t_{1}^{2} \cos ^{2}\left(k_{b} / 2\right)}{4 t_{1} t_{2} \cos \left(k_{b} / 2\right)}\right)
$$

and by taking into account that the angle of incidence is related to the group velocity $\partial E / \partial \boldsymbol{k}$ by means of

$$
\frac{v_{y}}{v_{x}}=\tan \theta_{i}=\frac{b}{a}\left(\frac{\tan \left(k_{b} / 2\right)}{\tan \left(k_{a} / 2\right)}+\frac{2 t_{1} \sin \left(k_{b} / 2\right)}{t_{2} \sin \left(k_{a} / 2\right)}\right) .
$$

These two equations allow to determine the two components of $\boldsymbol{k}_{i}$, as well as the pseudo-spin $\phi_{i}$ by means of (6).

The reflection and refraction laws of the electrons at the interface of the $p n$ junction are determined by the conservation of the electron energy $E$, the parallel component of the momentum $k_{\|}=-k_{x} \sin \alpha+k_{y} \cos \alpha$ and the normal component of the current density $j_{\perp}$ (due to the translational symmetry along interface and the continuity equation). These quantities are sketched in the kinematical construction in Figure 2 (c). The closed curves are constant energy contours in the regions I and

\footnotetext{
${ }^{2}$ We do not use here the expansion of the band structure around the $\Gamma$, because this causes already at relatively low energies discrepancies to the numerical simulations.
}

II. They have different size due to the electrostatic potential in region II. The straight blue-dashed line is given by $k_{y}=\left(k_{x}-k_{i, x}\right) \tan \alpha+k_{i, y}$ and represents the conservation of $k_{\|}$. Its intersection points with the constant energy contours determine the wave vectors $\boldsymbol{k}_{r / t}$ of the reflected and transmitted electrons. Note that the green semi-arcs indicate the regions where $j_{\perp}$ is conserved and hence, the correct solution for $\boldsymbol{k}_{t}$. With these wave vectors, we can calculate the pseudo-spins $\phi_{r}$ and $\phi_{t}$ from (6), as well as the angles of reflection $\theta_{r}$ and refraction $\theta_{t}$ using (10), which constitute the electron optics laws in phosphorene $p n$ junctions. An important consequence of the anisotropy of phosphorene's band structure is the fact that the current density (group velocity), momentum, and pseudo-spin are not always parallel. Therefore, the pseudo-spin angles $\phi$ and $\phi_{r}$ can not be interpreted as the angles of incidence and refraction respectively, as is usually the case for isotropic systems like graphene 63. This causes new and interesting phenomena to be discussed in Section V, which can be understood mainly by the fact that the pseudo-spin is conserved in the $p n$ junction as the electrostatic potential does not break the sublattice symmetry.

In order to obtain the probabilities of reflections $R$ and transmission $T$, we consider the wave function in region I

$$
\Psi_{\mathrm{I}}(\boldsymbol{r})=\frac{1}{\sqrt{2}}\left(\begin{array}{c}
1 \\
s \mathrm{e}^{\mathrm{i} \phi_{i}}
\end{array}\right) \mathrm{e}^{\mathrm{i} \boldsymbol{k}_{i} \cdot \boldsymbol{r}}+\frac{r}{\sqrt{2}}\left(\begin{array}{c}
1 \\
s \mathrm{e}^{\mathrm{i} \phi_{r}}
\end{array}\right) \mathrm{e}^{\mathrm{i} \boldsymbol{k}_{r} \cdot \boldsymbol{r}}
$$

and in region II

$$
\Psi_{\mathrm{II}}(\boldsymbol{r})=\frac{t}{\sqrt{2}}\left(\begin{array}{c}
1 \\
s^{\prime} \mathrm{e}^{\mathrm{i} \phi_{t}}
\end{array}\right) \mathrm{e}^{\mathrm{i} \boldsymbol{k}_{t} \cdot \boldsymbol{r}},
$$

where $s=\operatorname{sgn}(E)$ and $s^{\prime}=\operatorname{sgn}(E-V)$ are the band indices in the two regions. The amplitudes of reflection $r$ and transmission $t$ are calculated by using the continuity of the wave function at the interface. Finally, due to the conservation of the probability $T=1-|r|^{2}$, we obtain for the transmission probability

$$
T=\frac{2 \sin \left[\phi_{t}-\left(\phi_{r}+\phi_{i}\right) / 2\right] \sin \left[\left(\phi_{i}-\phi_{r}\right) / 2\right]}{s s^{\prime}-\cos \left(\phi_{t}-\phi_{r}\right)} .
$$

Note that the transmission is given as a function of the pseudo-spins, which in turn depend on the parameters $V, \alpha, E$ and $\theta$, see (6), 9) and (10). Hence, $T$ is an (implicit) function of these four parameters as we will demonstrate in Section $\nabla$

\section{THE NEGF METHOD FOR THE CURRENT FLOW}

Apart from the laws of reflection and refraction derived in the previous section, we will also calculate numerically the quantum coherent current flow in the phosphorene $p n$ junctions, starting from the tight-binding Hamiltonian of a finite system and applying the nonequilibrium Green's 

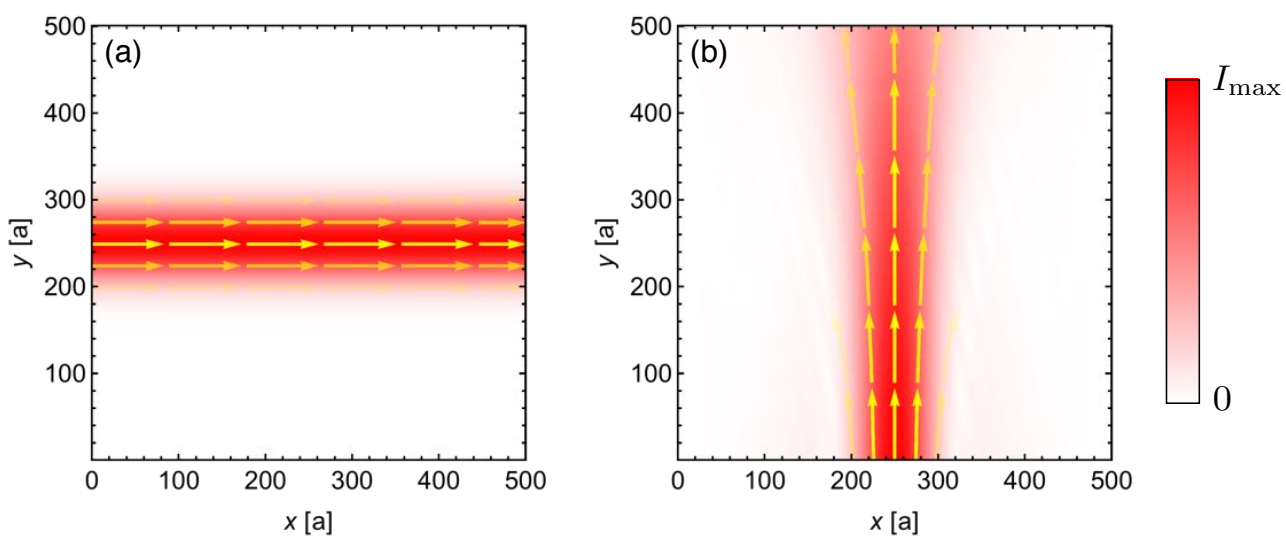

Figure 3. Current flow in a phosphorene nanoribbon without an electrostatic potential $(V=0)$. Electrons with energy $E=1.2\left|t_{1}\right|$ are injected at the left (a) and bottom (b) edge of the nanoribbon. The current density is indicated by the red color shading and its vector field by the yellow arrows. Ray-like ballistic electron propagation can be observed in both cases, although the diffraction of the electron beam is stronger in the $y$ direction (b) than in the $x$ direction (a).

function method (NEGF). As this method does not make use of the continuum approximation, it allows us to confirm numerically the electron optics laws. Moreover, wave effects like diffraction and interference are included which go beyond the ray-like trajectories of geometric optics.

In the following, we will summarize briefly the essential equations of the NEGF method. A detailed introduction can be found, for example, in Refs. 64, 65]. The Green's function of the system is given by

$$
G(E)=\left(E-H-\Sigma_{V}-\Sigma_{C}\right)^{-1}
$$

where $E$ is the energy of the electrons (times a unit matrix), $H$ is the tight-binding Hamiltonian (1) and $\Sigma_{V}=\sum_{n} V_{n}|n\rangle\langle n|$ the electrostatic potential with

$$
V_{n}= \begin{cases}0 & \text { if } d_{n} \leq-w(\text { region I) } \\ \left(d_{n} / w+1\right) V / 2 & \text { if }-w<d_{n}<w \text { (interface) } \\ V & \text { if } d_{n} \geq w \text { (region II) }\end{cases}
$$

where $d_{n}$ is the distance to the interface and $2 w \sim 5 a$ its width. Hence, we assume that on the atomic scale the junction has some smoothness (see below). In order to suppress boundary effects and mimic an infinite system, we place a constant complex potential $\Sigma_{C}=$ $-\mathrm{i} \sum_{n \in \text { edge }}|n\rangle\langle n|$ at the edges of the system, which absorbs the electrons.

The electrons are injected at the edges of the system as plane waves propagating towards the interface of the $p n$ junction. Their momentum $\boldsymbol{k}$, which in general does not indicate the direction of propagation, is calculated from the input parameters $E$ and $\theta$ using (9) and (10). The injection is represented by the inscattering function

$$
\Sigma_{S}^{\text {in }}=\sum_{n, m \in \text { edge }} A\left(\boldsymbol{r}_{n}\right) A\left(\boldsymbol{r}_{m}\right) \psi_{\boldsymbol{k}}^{*}\left(\boldsymbol{r}_{n}\right) \psi_{\boldsymbol{k}}\left(\boldsymbol{r}_{m}\right)|n\rangle\langle m|
$$

where the sum is over all atoms of the edge where the electrons are injected (for example, all atoms at the left edge in Figure $3(\mathrm{a}))$. The $\psi_{\boldsymbol{k}}\left(\boldsymbol{r}_{m}\right)$ are the eigenstates in (5) evaluated at the position $\boldsymbol{r}_{n}$ of the atoms at the edge. The function

$$
A(\boldsymbol{r})=\exp \left(-\left|\boldsymbol{r}-\boldsymbol{r}_{0}\right|^{2} / d_{0}^{2}\right)
$$

gives a Gaussian profile to the injected electron wave packet. The parameter $\boldsymbol{r}_{0}$ and $d_{0}$ control the position and width of the injected electron beam. Finally, the current flowing between the atoms at positions $\boldsymbol{r}_{n}$ and $\boldsymbol{r}_{m}$ is calculated by means of

$$
I_{n m}=\operatorname{Im}\left(t_{n m}^{*} G_{n m}^{\mathrm{in}}\right)
$$

where

$$
G^{\mathrm{in}}=G \Sigma_{S}^{\mathrm{in}} G^{\dagger} .
$$

Good agreement between the quantum current flow and the trajectories of geometric optics can be expected only in the specific parameter regime, where the Fermi wavelength of the electrons $\lambda_{F}$ is much larger than the interatomic distances $a, b$ and the junction width $w$ (justifying the approximations of the continuum and a sharp junction), but smaller than the system size $\left(L_{x}, L_{y}\right)$,

$$
a, b, w \ll \lambda_{F}=\frac{2 \pi}{|\boldsymbol{k}|} \ll L_{x}, L_{y} .
$$

In the following, we consider in our numerical studies a phosphorene nanoribbon of size $\left(L_{x}, L_{y}\right)=500 \times$ $500 a \approx 220 \times 220 \mathrm{~nm}$, which consists of approximately 1.4 million atoms. Electrons are injected at the energy $E=\Delta+0.2 t_{1} \approx 1.2\left|t_{1}\right| \approx 1.46 \mathrm{eV}$, which corresponds to a Fermi wavelength $\lambda_{F} \approx 12 a$. The width of the electron beam is $d_{0}=0.2 L_{x}$. The electron flow in absence of an electrostatic potential $(V=0)$ is shown in Figure 3 and confirms the ballistic, ray-like propagation of the electrons. Interestingly, we observe that the diffraction of the electrons is stronger in the $y$ direction 
(a)

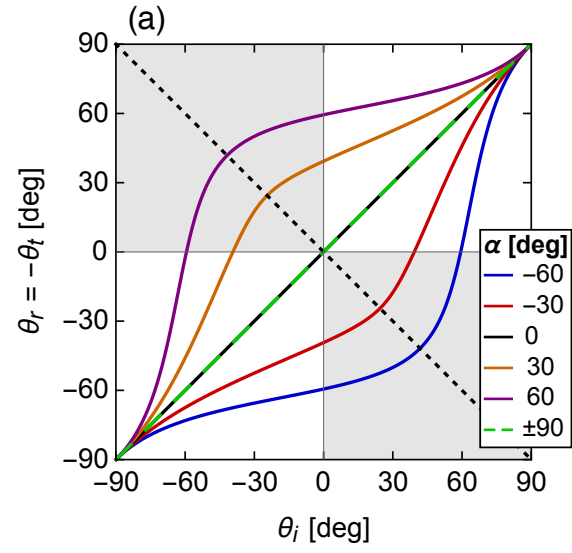

(b)

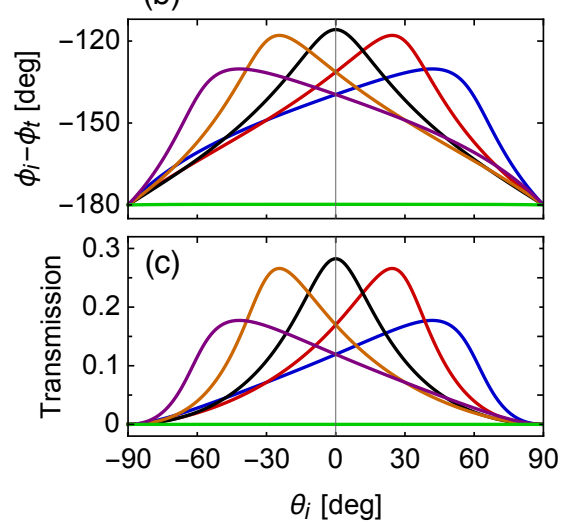

(d)

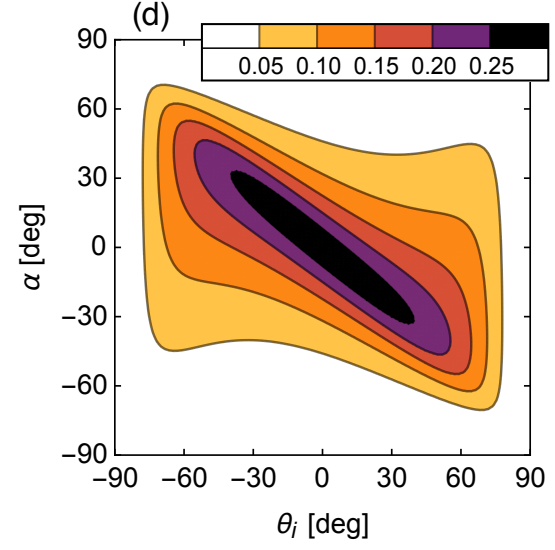

Figure 4. Electron optics laws in a phosphorene $p n$ junction with $V=2 E$ and electrons with energy $E=1.2\left|t_{1}\right|$. (a) Reflection angle $\theta_{r}$ as a function of the incidence angle $\theta_{i}$ for various tilting angles $\alpha$ of the junction. In general, atypical reflection $\left(\theta_{r} \neq \theta_{i}\right)$ is observed. The curves within the gray shaded squares indicate negative reflection. Typical reflection $\theta_{r}=\theta_{i}$ is found only if $\alpha=0^{\circ}, \pm 90^{\circ}$. The reflection laws are also valid for asymmetric junctions $(V \neq 2 E)$, but $\theta_{t}=-\theta_{r}$ holds only for the symmetric case. (b) Difference of the pseudo-spins for the incident and transmitted electrons, $\phi_{i}-\phi_{t}$. (c,d) Transmission as a function of $\theta_{i}$ and $\alpha$. Omnidirectional total reflection, called anti super-Klein tunneling, is observed in junctions with $\alpha= \pm 90^{\circ}$, because the pseudo-spins of the incident and transmitted electrons are anti-parallel. Note that the white regions in (d) correspond to a high degree of reflection.

(Schrödinger fermions) than in the $x$ direction (massive Dirac fermions). We will address this observation in our future work. From now on, we will measure all energies in multiples of $\left|t_{1}\right|$ and distances in multiples of $a$.

\section{NEGATIVE REFLECTION AND ANTI SUPER-KLEIN TUNNELING}

We evaluate the electron optics laws in phosphorene $p n$ junctions, which were derived in Section III, discuss their properties and compare with the current flow calculated numerically by the NEGF method. We consider a $p n$ junction with $V=2 E$, where the electrons go from the conduction band in region $I$ to the valence band in region II. The reflection angle $\theta_{r}$ as a function of the incidence angle $\theta_{i}$ is shown in Figure 4 (a) for various junction angles $\alpha$. The typical reflection law $\theta_{r}=\theta_{i}$ of optics is found only for junctions parallel to the $x\left(\alpha=0^{\circ}\right)$ and $y$ axes $\left(\alpha= \pm 90^{\circ}\right)$, but in general $\theta_{r}$ is a non-linear function of $\theta_{i}$.

Remarkably, we find negative reflection for certain parameters, see the curves within the gray shaded regions in Figure 4 (a). Negative reflection is observed in tilted junctions for incidence angles $0<\left|\theta_{i}\right|<\left|\theta_{M}\right|$, where $\theta_{M}$ is the incidence angle for normal reflection, see the intersection points of the curves in Figure 4 (a) with the horizontal $\theta_{r}=0^{\circ}$ line. For $\left|\theta_{i}\right|>\left|\theta_{M}\right|$, anomalous reflection is observed, because $\theta_{i}$ and $\theta_{r}$ have the same sign but different values. Moreover, retroreflection $\theta_{r}=-\theta_{i}$ emerges for certain incidence angles, see the intersection points of the curves in Figure 4 (a) with the black-dotted line. Note that these atypical reflection laws in phosphorene $p n$ junctions are independent of $V$ and hence also valid for junctions with $V \neq 2 E$. In symmetric junctions $(V=2 E)$, we can also confirm numerically that $\theta_{r}=-\theta_{t}$ and hence, negative reflection goes along with positive refraction and vice versa.

All these unusual properties are confirmed in Figure 5 which shows the current flow paths calculated numerically by the NEGF method together with the trajectories from geometric optics. The upper panels (a-c) show three cases of normal incidence but the different orientations of the junction causes that the reflection and refraction of the electrons are very different. Typical (specular) reflection is observed in the panels $(\mathrm{d}, \mathrm{e})$, while negative reflection is found in panel (f). Note that the absorption of the electrons at the edges due to the complex potential is not perfect and hence, typical reflection is observed at the edges. The numerically calculated current densities show in some cases a ripple pattern, which is on the length scale of the Fermi wavelength and caused by the interference of electron beams of finite width.

The observed atypical reflection law can be explained by the anisotropy of phosphorene's electronic structure and the tilting of the junction. Due to these, the intersection points of the blue-dashed line in Figure 2 (c) with the constant energy contours represent states having different parallel group velocity component, although the linear momentum along the interface is conserved. Only if $\alpha=0^{\circ}, \pm 90^{\circ}$, the intersection points represent states with the same parallel group velocity component causing typical reflection. Note that the atypical reflection can also be explained by the fact that the tilting of the junction breaks the mirror symmetry with respect to the $k_{x}$ and $k_{y}$ axes in momentum space.

The transmission shown in Figure 4 (c-d) changes with the tilting of the junction $\alpha$. Klein tunneling, one of the 
(a)

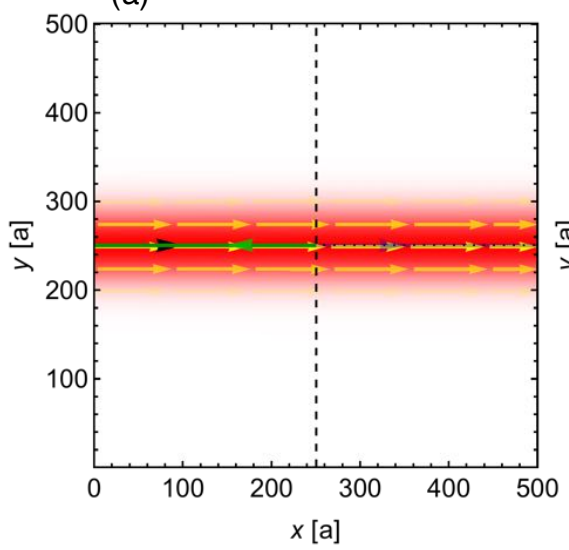

(d)

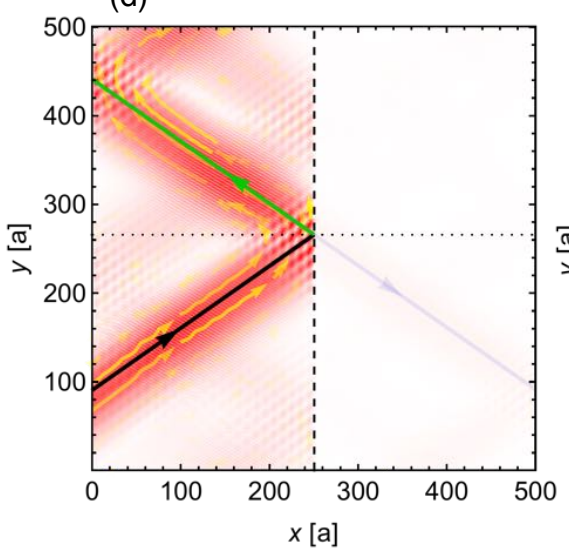

(b)

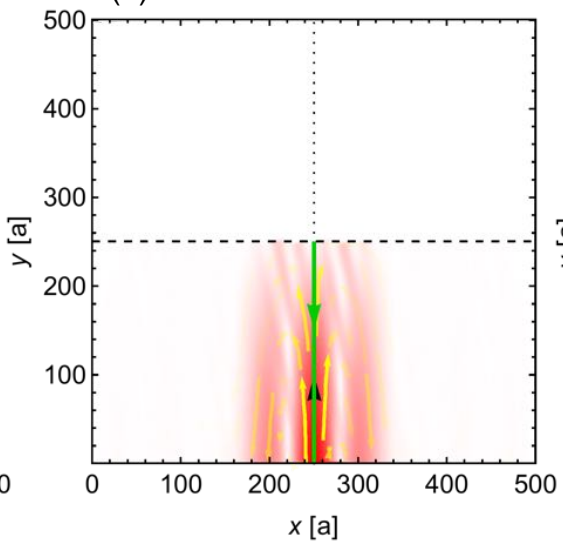

(e)

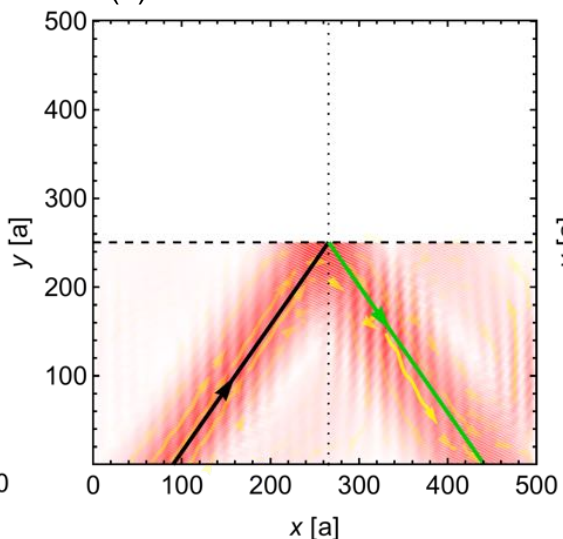

(c)

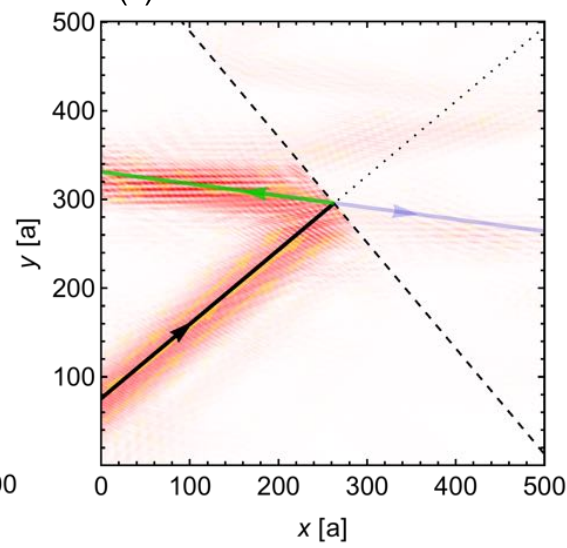

(f)

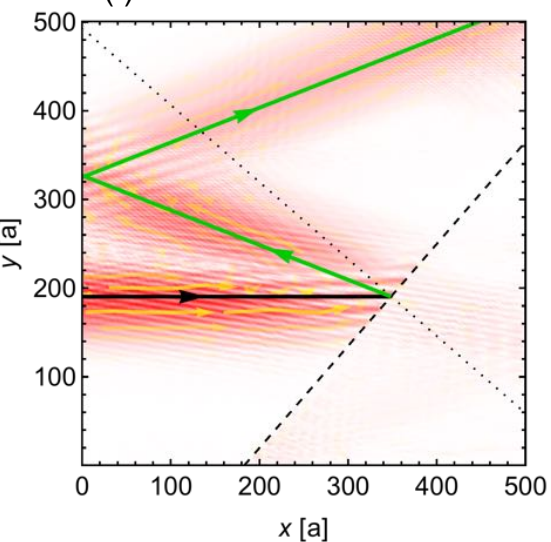

Figure 5. Current flow in a phosphorene $p n$ junction with $V=2 E$ and $E=1.2\left|t_{1}\right|$ for different tiltings of the junction. The interface of the junction is indicated by a black-dashed line and its normal by a black-dotted line. The numerically calculated current flow paths (the red color shading indicates the current density, the yellow arrows the current vector field), agree qualitatively with the ray-like trajectories (solid lines) of geometric optics and hence, confirm the electron optics laws derived in Section III. (a-c) Three different cases of normal incidence show that the reflection and refraction of the electron beams depend strongly on the tilting $\alpha$. (d,e) Typical (specular) reflection is found only if $\alpha=0^{\circ}$, $\pm 90^{\circ}$. (f) Negative reflection of the electron beam is observed at the interface of the junction, while typical reflection is found at the the edges of the system. (b,e) For junctions with $\alpha= \pm 90^{\circ}$, we observe omni-directional total reflection, called anti super-Klein tunneling, which is due to the fact that the pseudo-spins of the incident and transmitted electrons are anti-parallel.

outstanding properties of graphene [6, 7], is not observed in phosphorene, because $T<1$. More notably, we observe zero transmission in a junction with $\alpha= \pm 90^{\circ}$ for all incidence angles. This omni-directional total reflection, called anti-super Klein tunneling, is confirmed numerically in Figure 5 (b,e). In contrast to the typical total reflection, it is not caused by the absence of electronic states but can be explained by the fact that the pseudo-spins of the incident and transmitted electrons are anti-parallel. The absence of Klein tunneling is also due to these pseudo-spin differences $\phi_{i}-\phi_{t}$ shown in Figure 4 (b). Interestingly, the difference is quite similar to the transmission, though an exact scaling law cannot be established. Note that phosphorene's anisotropic energy bands and the tilting of the $p n$ junction can also cause asymmetric Veselago lenses, such as those predicted for uniaxially strained graphene 12 .
In asymmetric phosphorene $p n$ junctions, where $V \neq$ $2 E$, further interesting electron-optics phenomena can be observed. In Figure 6 the electrostatic potential is increased to higher energies $V \sim 3.5\left|t_{1}\right|$, while the electron energy $E=1.2\left|t_{1}\right|$ is kept constant. We observe that the electrons are refracted in a very narrow window, $\left|\theta_{t}\right| \lesssim 1^{\circ}$, compared to the broad range of incident angles ranging from $-90^{\circ}$ to $90^{\circ}$. The transmission is decaying almost linearly from $T \approx 0.5$ at normal incidence to zero at $\theta_{i}= \pm 90^{\circ}$, see Figure 6 (b). Hence, the $p n$ junction acts a collimator and a filter, because the transmitted electrons are aligned to flow all in the same direction and the normally incident electrons are transmitted preferentially. Such collimation effect can be obtained also in a graphene superlattice [27] but not in a single graphene $p n$ junction. The numerically calculated current flow in Figure $6(\mathrm{c}, \mathrm{d})$ confirms these effects. A narrow electron 
(a)

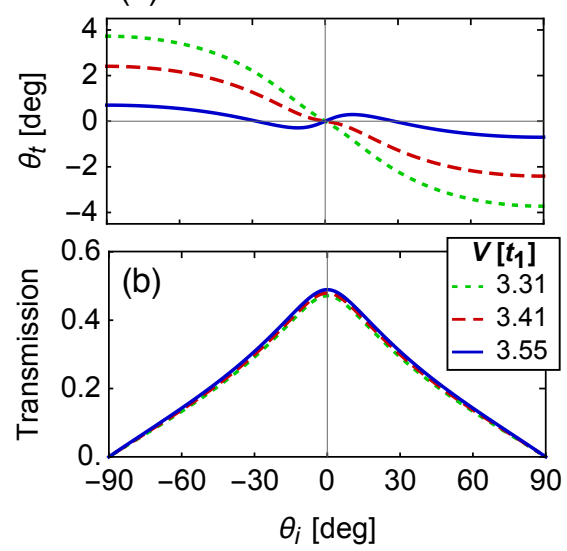

(c)

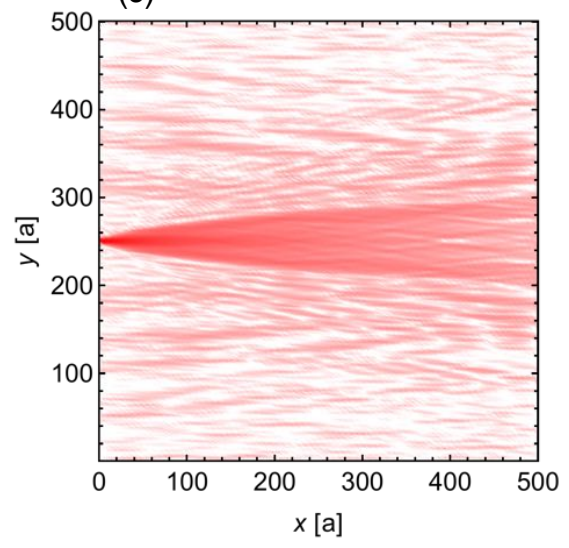

(d)

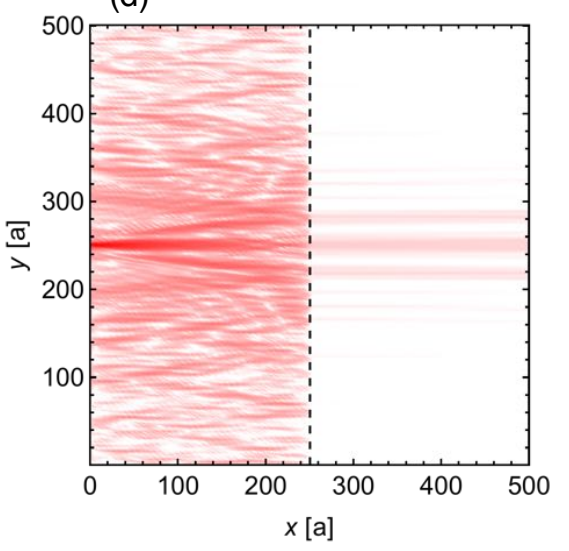

Figure 6. Collimation of electrons in phosphorene $p n$ junctions. (a) Refraction angle as a function of the incidence angle for electrons with energy $E=1.2\left|t_{1}\right|$ and different values of the electrostatic potential $V$. (b) Transmission as a function of the incidence angle. (c,d) Numerically calculated current flow for a narrow electron beam in absence (c) and presence (d) of the electrostatic potential. Note that a logarithmic scale is used for the red color shading of the current density, because the diffraction naturally reduces its density. All panels show that a phosphorene $p n$ junction can act as a collimator, because the electrons are transmitted all in the same direction normal to the junction. It also acts as a filter because normally incident electrons are transmitted preferentially.

beam is injected at the left system edge. In absence of the electrostatic potential it shows strong diffraction (c), while in presence of the potential the collimation and filtering of the electrons can be observed clearly (d).

\section{CONCLUSIONS}

The ballistic electron flow in phosphorene $p n$ junctions has been studied theoretically. We have started with a second nearest neighbor tight-binding Hamiltonian that captures the essential features of phosphorene's electronic structure at low energies, namely a band gap and strong anisotropy. Due to this anisotropy the electrons behave in one direction as massive Dirac fermions and in the orthogonal direction as Schrödinger electrons, leading to unusual transport properties.

Applying the continuum approximation, we have derived the electron optics laws in phosphorene which show very particular properties. Because of the anisotropy of the electronic structure, the orientation of the $p n$ junction with respect to the sublattice is an important parameter, see Figure 2. For tilted junctions $0^{\circ}<|\alpha|<90^{\circ}$, we find negative and anomalous reflection, where $\theta_{r} \neq \theta_{i}$, see Figure 4. Such atypical properties have been observed for the reflection of acoustic and electromagnetic waves at the surface of metamaterials [55 58, but - to the best of our knowledge - not yet for the electron flow in a nano-material. The typical reflection law $\theta_{r}=\theta_{i}$ is obtained only for junctions aligned with the $x$ or $y$ axis of the system. Moreover, for junctions parallel to the $y$ axis, we observe omni-directional total reflection, called anti-super Klein tunneling, which is explained by the fact that the pseudo-spins are anti-parallel in the two regions of the junction. This effect is the counterpart of the omni-directional perfect transmission of massless Dirac fermions in Dirac materials [17 and pseudo-spin one systems 5962 . We have also applied the NEGF method on the tight-binding model to calculate numerically the current flow in finite phosphorene nanoribbons. The good agreement of both approaches confirms clearly the electron optics laws in phosphorene, see Figure 5 , but also reveals additional interference effects due to the wave nature of the electron beams.

The exiting transport properties reported in this work will certainly help to pave the way to the first electron optics experiments in phosphorene $p n$ junctions, but may also have various nano-technological applications. It has been shown in Figure 6 that the anisotropy of the electronic structure can be used to construct a collimator and filter of electron beams. The anti-super Klein tunneling can be used to confine and guide efficiently an electron beam, or to switch its direction by turning on and off the electrostatic potential.

\section{ACKNOWLEDGMENTS}

The authors gratefully acknowledge financial support from CONACYT Proyecto Fronteras 952 and the UNAM-PAPIIT research grants IN-103017 and IA101618. We thank Reyes Garcia for computer technical support and Thomas H. Seligman for helpful discussions. 
[1] G. Pozzi, Particles and Waves in Electron Optics and Microscopy (Academic Press, 2016).

[2] V. V. Cheianov, V. Fal'ko, and B. L. Altshuler, Science 315, 1252 (2007).

[3] J. R. Williams, T. Low, M. S. Lundstrom, and C. M. Marcus, Nat. Nanotechnol. 6, 222 (2011).

[4] P. Rickhaus, P. Makk, K. Richter, and C. Schönenberger, Appl. Phys. Lett. 107, 251901 (2015).

[5] P. Rickhaus, M.-H. Liu, P. Makk, R. Maurand, S. Hess, S. Zihlmann, M. Weiss, K. Richter, and C. Schönenberger, Nano Lett. 15, 5819 (2015).

[6] M. I. Katsnelson, K. S. Novoselov, and A. K. Geim, Nat. Phys. 2, 620 (2006).

[7] A. F. Young and P. Kim, Nat. Phys. 5, 222 (2009).

[8] G.-H. Lee, G.-H. Park, and H.-J. Lee, Nat. Phys. 11, 925 (2015).

[9] S. Chen, Z. Han, M. M. Elahi, K. M. M. Habib, L. Wang, B. Wen, Y. Gao, T. Taniguchi, K. Watanabe, J. Hone, et al., Science 353, 1522 (2016).

[10] T. Stegmann and N. Szpak, New J. Phys. 18, 053016 (2016).

[11] T. Stegmann and N. Szpak, 2D Mater. 6, 015024 (2019).

[12] Y. Betancur-Ocampo, Phys. Rev. B 98, 205421 (2018).

[13] G. G. Naumis, S. Barraza-Lopez, M. Oliva-Leyva, and H. Terrones, Rep. Prog. Phys. 80, 096501 (2017).

[14] M. Oliva-Leyva and G. G. Naumis, Phys. Rev. B 88, 085430 (2013).

[15] R. Carrillo-Bastos, C. León, D. Faria, A. Latgé, E. Y. Andrei, and N. Sandler, Phys. Rev. B 94, 125422 (2016).

[16] E. Andrade, R. Carrillo-Bastos, and G. G. Naumis, Phys. Rev. B 99, 035411 (2019).

[17] Y. Betancur-Ocampo, J. Phys.: Condens. Matter 30, 435302 (2018).

[18] J. L. Garcia-Pomar, A. Cortijo, and M. Nieto-Vesperinas, Phys. Rev. Lett. 100, 236801 (2008).

[19] Z. Wu, F. Zhai, F. M. Peeters, H. Q. Xu, and K. Chang, Phys. Rev. Lett. 106, 176802 (2011).

[20] F. Zhai, Y. Ma, and K. Chang, New J. Phys. 13, 083029 (2011).

[21] M. M. Grujić, M. Ž. Tadić, and F. M. Peeters, Phys. Rev. Lett. 113, 046601 (2014).

[22] V. H. Nguyen, S. Dechamps, P. Dollfus, and J.-C. Charlier, Phys. Rev. Lett. 117, 247702 (2016).

[23] M. Settnes, S. R. Power, M. Brandbyge, and A.-P. Jauho, Phys. Rev. Lett. 117, 276801 (2016).

[24] B. Sa, Y.-L. Li, J. Qi, R. Ahuja, and Z. Sun, J. Phys. Chem. C 118, 26560 (2014).

[25] D. Zhai and N. Sandler, Phys. Rev. B 98, 165437 (2018).

[26] R. Carrillo-Bastos, M. Ochoa, S. A. Zavala, and F. Mireles, Phys. Rev. B 98, 165436 (2018).

[27] C.-H. Park, Y.-W. Son, L. Yang, M. L. Cohen, and S. G. Louie, Nano Lett. 8, 2920 (2008).

[28] L. Li, Y. Yu, G. J. Ye, Q. Ge, X. Ou, H. Wu, D. Feng, X. H. Chen, and Y. Zhang, Nat. Nanotechnol. 9, 372 (2014).

[29] M. C. Watts, L. Picco, F. S. Russell-Pavier, P. L. Cullen, T. S. Miller, S. P. Bartuś, O. D. Payton, N. T. Skipper, V. Tileli, and C. A. Howard, Nature 568, 216 (2019).

[30] L. Li, J. Kim, C. Jin, G. J. Ye, D. Y. Qiu, F. H. da Jornada, Z. Shi, L. Chen, Z. Zhang, F. Yang, et al., Nat. Nanotechnol. 12, 21 (2016).
[31] A. Carvalho, M. Wang, X. Zhu, A. S. Rodin, H. Su, and A. H. C. Neto, Nat. Rev. Mater. 1, 16061 (2016).

[32] L. Kou, C. Chen, and S. C. Smith, J. Phys. Chem. Lett. 6, 2794 (2015).

[33] N. Ehlen, B. V. Senkovskiy, A. V. Fedorov, A. Perucchi, P. D. Pietro, A. Sanna, G. Profeta, L. Petaccia, and A. Grneis, Phys. Rev. B 94, 245410 (2016).

[34] X. Peng, Q. Wei, and A. Copple, Phys. Rev. B 90, 085402 (2014).

[35] E. T. Sisakht, M. H. Zare, and F. Fazileh, Phys. Rev. B 91, 085409 (2015).

[36] E. T. Sisakht, F. Fazileh, M. H. Zare, M. Zarenia, and F. M. Peeters, Phys. Rev. B 94, 085417 (2016).

[37] M. Ezawa, New J. Phys. 16, 115004 (2014).

[38] D. Midtvedt, C. H. Lewenkopf, and A. Croy, J. Phys.: Condens. Matter 29, 185702 (2017).

[39] L. C. L. Y. Voon, A. Lopez-Bezanilla, J. Wang, Y. Zhang, and M. Willatzen, New J. Phys. 17, 025004 (2015).

[40] A. Brown and S. Rundqvist, Acta Crystallogr. 19, 684 (1965).

[41] A. N. Rudenko and M. I. Katsnelson, Phys. Rev. B 89, 201408(R) (2014).

[42] A. N. Rudenko, S. Yuan, and M. I. Katsnelson, Phys. Rev. B 92, 085419 (2015).

[43] D. Çakır, H. Sahin, and F. M. Peeters, Phys. Rev. B 90, 205421 (2014).

[44] M. Elahi, K. Khaliji, S. M. Tabatabaei, M. Pourfath, and R. Asgari, Phys. Rev. B 91, 115412 (2015).

[45] L. L. Li, B. Partoens, and F. M. Peeters, Phys. Rev. B 97, 155424 (2018).

[46] W.-J. Zhu, W. Zhao, and J.-W. Ding, J. Phys.: Condens. Matter 30, 33LT01 (2018).

[47] S. P. Koenig, R. A. Doganov, H. Schmidt, A. H. C. Neto, and B. Özyilmaz, Appl. Phys. Lett. 104, 103106 (2014).

[48] Z.-Y. Ong, Y. Cai, G. Zhang, and Y.-W. Zhang, J. Phys. Chem. C 118, 25272 (2014).

[49] S. D. Sarkar, A. Agarwal, and K. Sengupta, J. Phys.: Condens. Matter 29, 285601 (2017).

[50] J. Miao, L. Zhang, and C. Wang, 2D Mater. 6, 032003 (2019).

[51] H. Y. Lv, W. J. Lu, D. F. Shao, and Y. P. Sun, Phys. Rev. B 90, 085433 (2014).

[52] M. Mehboudi, K. Utt, H. Terrones, E. O. Harriss, A. A. P. SanJuan, and S. Barraza-Lopez, PNAS 112, 5888 (2015).

[53] Z. Naemi, M. J. Tafreshi, N. Salami, and A. Shokri, J. Mater. Sci. 54, 7728 (2019).

[54] A. Chaudhury, S. Majumder, and S. J. Ray, Phys. Rev. Appl. 11, 024056 (2019).

[55] B. Liu, W. Zhao, and Y. Jiang, Sci. Rep. 6, 38314 (2016).

[56] S. Liu, T. J. Cui, A. Noor, Z. Tao, H. C. Zhang, G. D. Bai, Y. Yang, and X. Y. Zhou, Light Sci. Appl. 7, 18008 (2018).

[57] N. Yu, P. Genevet, M. A. Kats, F. Aieta, J.-P. Tetienne, F. Capasso, and Z. Gaburro, Science 334, 333 (2011).

[58] N. Yu and F. Capasso, Nat. Mater. 13, 139 (2014).

[59] D. F. Urban, D. Bercioux, M. Wimmer, and W. Häusler, Phys. Rev. B 84, 115136 (2011).

[60] R. Shen, L. B. Shao, B. Wang, and D. Y. Xing, Phys. Rev. B 81, 041410(R) (2010). 
[61] Y. Betancur-Ocampo, G. Cordourier-Maruri, V. Gupta, and R. de Coss, Phys. Rev. B 96, 024304 (2017).

[62] A. Fang, Z. Q. Zhang, S. G. Louie, and C. T. Chan, Phys. Rev. B 93, 035422 (2016).

[63] P. E. Allain and J. N. Fuchs, Eur. Phys. J. B 83, 301 (2011).
[64] S. Datta, Electronic Transport in Mesoscopic Systems (Cambridge University Press, 1995).

[65] S. Datta, Quantum Transport: Atom to Transistor (Cambridge University Press, 2005). 